\title{
Concepts for Analysis and Design of Mobile Healthcare Applications
}

\author{
Joseph McKnight ${ }^{1}$, Gavin Doherty ${ }^{1}$, Bridget Kane ${ }^{1,2}$, and Saturnino Luz ${ }^{1}$ \\ ${ }^{1}$ Department of Computer Science, Trinity College Dublin, Dublin 2, Ireland \\ ${ }^{2}$ Trinity Centre for Health Sciences, St. James's Hospital, Dublin
}

\begin{abstract}
In complex domains such as healthcare, careful analysis of user requirements is an important aspect of the development process. In recent years, ethnographic study has become a popular tool for building up an understanding of the healthcare domain. However, linking observational data with the design and development process is a challenging problem. A range of conceptual frameworks have been proposed which can aid in transforming these data into concrete requirements. In this paper, we argue that the framework and associated design concepts used will have a strong influence on shaping the outcome of design, and that the development team should consider carefully which are most appropriate to the problem they face. We use a case study based around a patient review process as an illustrative example.
\end{abstract}

Keywords: Conceptual Analysis, Concepts, Healthcare, Ethnographic Study, Mobility.

\section{Introduction}

The healthcare environment raises many challenges for design, with many different roles and stakeholders involved, safety critical tasks being performed, large volumes of information, and highly mobile workers carrying out their activities in a variety of different settings. Standard user-centered design processes can be difficult to apply to such complex work environments, and while techniques such as participatory design are useful they are not going to fully address the problem [7].

In recent years, ethnographic studies have become a popular tool for getting to grips with the complexity of the healthcare environment, with a number of projects producing ethnographically-informed designs. Take-up has been such that commercially driven initiatives are now making use of these techniques. Typically, an observational investigation will yield a lot of information on the different users, working practices, activities as they currently happen, use of artefacts and information. However such studies do not necessarily produce actual design guidance, and in particular they are not a sufficient basis for reasoning about the effects of changes on the work, the workers, and the environment. These are dynamic environments, and are subject to many external factors, including evolving "best practice", changing regulations and organizational structures, and continuous upgrading of information systems. 
A number of different analytic frameworks have been proposed, which can help to transform this ethnographically derived information into input to the design process. Hence teams looking at the development of such information systems are faced with the choice of which framework to use. In the following sections we argue that this choice will have a strong effect on shaping the designs which are produced, and will also impact on the ability to reason about evolution of the overall system in response to higher level changes within the organisation (for example, the push towards multidisciplinary team meetings). The concepts considered in this paper have previously been applied to the healthcare environment and due to the nature of the environment many of these have an explicitly mobile aspect.

\section{Case Study - Patient Case Review}

The case study concerns a large tertiary referral and cancer treatment healthcare facility, where the cardio-thoracic surgery unit receives 5 to 10 new case referrals per week. Each new referral must be processed in order to determine the patient's suitability for surgery. This involves gathering the information needed to discuss the patient at a multi-disciplinary team meeting (MDTM), which stages (classifies) the patient's cancer, followed by an outpatient assessment before deciding if surgery is required, followed then by the surgery itself and aftercare. Given the large number of specialist staff that collaborate through the MDTM, it is vital that the necessary tests have been carried out and reviewed prior to the MDTM. The work of managing the patients through the surgical process is mainly carried out by the consultant cardiothoracic surgeon and specialist oncology coordinator nurse (hereafter referred to as the coordinator), aided by administrative staff.

The processing of patients is tracked by various paper based artefacts that are maintained by the coordinator. The artefacts used include paper notebooks with the most relevant patient information, lists of patients in treatment with status, and a file with all documents related to the patient. This is necessary because typically patients in process are not physically located at the hospital ward and may not technically be a patient of the hospital until outpatient assessment or surgery. Even though patient treatment is managed from the tertiary referral hospital, scans and tests may be carried out by proxy at the referring hospital. As a result, the system is highly dependent on the coordinator and paper based artefacts to function. For example, a patient's processing may be on hold until a scan is completed at another hospital or a test result becomes available. Unless these actions are followed up by the coordinator, using paper files, notebook and various artefacts, a delay in processing could result, with potentially serious consequences. While the use of a paper-based system may seem atypical, the constant evolution of processes means that often workers have to fall back on ad-hoc methods while they wait for formal structures and technological support to emerge.

In order to investigate requirements prior to the introduction of electronic support for these activities, an ethnographic study was performed with the relevant clinicians and administrative staff at the hospital. This study is based on semi-structured interviews with clinicians, examination of paper-based artefacts, and observations of a number of key locations within the working environment. A previous and highly 
detailed ethnographic study of the MDTM was also of much benefit in understanding these activities (Kane and Luz, 2006).

\section{The patient review process}

When a patient is referred to the cardio-thoracic surgeon a letter of referral is received at the tertiary referral hospital and transferred to the coordinator for the cardiothoracic unit. The coordinator will record the patients' details to a note book and store the letter of referral along with any other documents received to a file, which is then placed in a portable carry case. The patients' case will not be discussed again until the weekly meeting of the coordinator and surgeon. Current and new patients are discussed at this meeting in order to decide on tests that are needed before the patients case can be discussed at an MDTM. The coordinator records the tests required and follows up on the actions required with the patient and referring hospital to ensure they are carried out. Until these actions have been completed the patient is not yet 'active': these tests must be completed before deciding if the patient is ready to be discussed at the MDTM. Scans carried out at other hospitals will be forwarded to the tertiary referral hospital to be examined by the specialist radiologist.

When this initial processing is complete the patients are put on a list for discussion at a weekly MDTM, or referred back to the initial process if more information is needed. The coordinator is responsible for ensuring that the patient names are on the MDTM list and that the patient information needed at the MDTM is available. The surgeon discusses the patient cases with other clinicians at the MDTM, while the coordinator records follow up actions needed and decisions made. Once again the coordinator is responsible to implement follow up actions needed.

The patients that have been recommended for surgery at the MDTM come to the hospital for an outpatient assessment of general health before a final decision on surgery is made. While the assessment is carried out by a member of the surgical team the coordinator records the information on a self made artefact. The patients assessed will then have their cases discussed at the next meeting between the coordinator and surgeon. The coordinator will have to manage the patients requiring discussion after the outpatient assessment and weekly meeting, along with other follow up actions. In addition to the work described above in processing patients there are other tasks that need to be carried out. For instance the coordinator must answer queries on information related to patient processing from patients themselves, other hospitals, and colleagues. From the patient processing information the surgeon is mainly interested in the numbers in process, at each phase, awaiting MDTM, outpatient assessment and surgery.

\section{Design Frameworks}

We consider in this section concepts which have been used in ethnographically informed healthcare case studies on hospital wards, with a view to informing design. It is important to note that while these frameworks are not orthogonal, each contributes a unique conceptual lens through which the ethnographic data is analysed and interpreted. We briefly consider the relevance of each framework to the case study, and the degree to which they can shape design activities. 
Table 1. Concepts and Abstractions used in Analysis and Design in Healthcare

\begin{tabular}{ll}
\hline Concept & Purpose \\
\hline Rhythms & $\begin{array}{l}\text { Conceptual framework to explore relationship between information } \\
\text { seeking and temporal coordination. } \\
\text { Conceptual framework to explore effects of moving people and things } \\
\text { (artefacts, equipment) to accomplish work. }\end{array}$ \\
$\begin{array}{l}\text { Common Information } \\
\text { Conceptual framework for analysis of a hospital CIS to reveal issues that } \\
\text { Cognitive Artefacts }\end{array}$ & $\begin{array}{l}\text { An approach to uncover and understand the cognitive work in healthcare } \\
\text { for the design of digital artefacts. }\end{array}$ \\
Coordinative Artefacts & $\begin{array}{l}\text { To understand how coordination and cooperation of workers on a ward is } \\
\text { enabled through a network of artefacts. } \\
\text { Abstraction that bases support systems around main activities that clini- } \\
\text { cians perform daily. }\end{array}$ \\
\hline Computing &
\end{tabular}

\section{Temporal Rhythms}

The concept of temporal rhythms (repeating daily patterns of work) is used [11] to analyse the information seeking activities of clinicians working on a ward. These typical working rhythms include shift change, morning rounds, medication, meetings and new arrivals etc, with each rhythm necessitating different information seeking activities. For example, a clinician might order a lab test knowing they will need the results for the following morning rounds; a nurse finishing a shift prepares all the information required for the handover. The rhythms concept leads us to concentrate on the information seeking and providing activities of clinicians that are a part of these working rhythms.

Although working rhythms in the case study are not occurring on a daily basis the concept still proves useful in analysing the information seeking and providing activities of the clinicians. The patient case discussion is the central provider and seeking rhythm in the system. The information sought in this patient case discussion process is provided to the MDTM. The MDTM often also acts as a source of information for future case discussions. This reciprocal relationship is also true between the case discussions and outpatient assessment.

\section{Mobility Work}

The concept of mobility work is used by [3] to describe the spatial aspect of cooperative work that is necessary for clinicians to accomplish tasks on a hospital ward. This entails that the correct configuration of people,places, resources and knowledge to be achieved to accomplish a task, while operating in an environment where these resources are also mobile. In general terms, mobility work is the work that must be performed so that clinicians can carry out tasks at specific locations.

Analysis of the case study reveals multiple resources required at the MDTM, outpatient and angiogram rooms to support task accomplishment. MDTM and PTF equipment are fixed to the room location where they are needed. However other resources are mobile such as patient files and notebooks, while the radiology scans and reports are available where network or PC access is present. As only the patients who must have their case discussed at the next MDTM or angiogram room meetings are 
required, the coordinator must organise this information ahead of the meetings. This involves creating a list of patients to be discussed while ensuring that other relevant information is also ready. Overall, the concept was found to be applicable to our case study, and suggests increased support for mobilisation of resources.

\section{Common Information Space}

The concept of a common information space (CIS) [2] is used by [13] to analyse cooperative work of heterogeneous workers on an ICU ward, which is based on the use of a common information repository, HealthStat. Their observations revealed a number of issues, firstly that physical proximity of co-workers does not equate to mutual understanding to enable smooth coordination and interpretation of each other's work; this was not the case in other studies of a hospital CIS [5]. Secondly, those heterogeneous workers have a different representation of the underlying information stored in HealthStat, so coordinating activities relies on each representation reflecting accurate shared data, with any change propagated to each representation.

An obvious issue identified from our CIS analysis is the reliance on the coordinator to relay information verbally to the consultant when discussing cases due to access restrictions to paper artefacts. The consultant must perform a similar CIS transfer at the MDTM, and is able to use the HIS to show scans and reports, but must refer to the coordinator for non medical processing information. This temporary immersion and information transfer in a "foreign" CIS for the duration of a task could be supported by mobile devices that facilitate shared views on data. While there are many potential issues surrounding the introduction of such technology [1], the framework is found to be relevant to the case study and design.

\section{Cognitive Artefacts}

The failure of automation in healthcare to improve clinical performance is examined by [14] who suggest that this is due to the design concepts on which these systems are based. Current healthcare displays do not represent the underlying domain semantics [12] and therefore are not suited to assisting clinicians in the cognitive work that they must perform, which forces them to perform extra work to overcome these deficiencies. To design displays that support clinicians in the work they actually perform requires a significant investigation of the technical work [10], i.e. non clinical work, that is performed in order to enable clinical work to happen. One way of uncovering this technical work is creation and usage of cognitive artefacts [9].

The cognitive artefacts concept focuses investigation on the artefacts that are created by clinicians so that we can uncover the work that the artefact is designed to support. This will reveal the goals and strategies employed by the artefact users during their work. Investigation of the work the artefact supports will ensure that any digital replacement is created with an understanding of how work is managed using the artefact, and provide appropriate support. It is important to perform such analysis as simply mimicking a paper based artefact may not equate to supporting the work it was designed to help.

The coordinator in our case study creates patient lists to act as external representations of the patients. These are not annotated or stored, but guide case discussions. A digital replacement could be created to support this task. This could involve the coordinator dynamically managing these lists using a mobile device in situ as required instead of en-masse prior to meetings. The coordinator also uses a notebook to record 
summary patient processing information such as scans, tests and patients status for quick reference. This is also used during case discussions and MDT meetings. Digitising this notebook requires an understanding of these multiple roles it plays and the work processes it is used within. As patient lists are created from and are a subset of the notebook information, its electronic counterpart should support this creation and migration of information. The recorded scans, tests and reports in the notebook could also be linked to the HIS, which could track and update patient processing status automatically, instead of requiring a search for patient details on the HIS and recording them to the notebook. Again, we can see that the framework is relevant to the case study, with a particular focus to the type of support suggested.

\section{Activity Based Computing}

The concept of Activity-Based Computing (ABC) is explored by [6] to investigate providing support for healthcare work. The activity abstraction is used due to concerns about the suitability of traditional paradigms, such as application and document centered systems, to an environment where work is "nomadic, collaborative, intensive and often interrupted". ABC is designed to allow activities to be suspended and resumed when interrupted and handed over to colleagues to support ad-hoc collaboration.

Activity Based Computing was proposed as an alternative to document and application centred system due to the nomadic, collaborative intensive and often interrupted nature of work in healthcare. While activity management is an important aspect of the work, and the issues targeted by the framework (collaborative, intensive, nomadic, interruptions) are relevant, the framework was found to apply more to the wider context of the application, rather than design for the review process itself.

\section{Coordinative Artefacts}

Bardram and Bossen [4] look at coordination and collaboration on a hospital ward by analysing usage of non digital coordinative artefacts. It was found that these artefacts (worksheets, whiteboards etc) facilitated locating patients and staff, cooperative planning, continuous coordination, status overview and passing messages. This has implications for development that digitises these artefacts as the functionality afforded to clinicians must be retained.

The study in [4] was focused on a heterogeneous group of workers on a hospital ward and is not directly applicable to our case study as the non digital artefacts created by our clinicians are primarily created for their own personal use, with some minor exceptions. While it is possible that the work system could well benefit from making greater use of such artefacts, it was not found to be immediately applicable to the case study.

\section{Future Work}

The question of how to choose an appropriate framework has not yet been fully addressed. We would argue that the closer the symmetry between the chosen concept and work system under study, the easier it will be to elucidate and communicate requirements for design. Specifically we need to address what constitutes a "fit" between a work system and concept and how we can extract requirements or design recommendations from analysis. 
While each concept will prove more or less applicable to any given work system, the range of relevant design concepts goes beyond those considered in this paper. It could be that the process of attempting to apply a number of concepts to analysis of a particular setting may facilitate selection or development of a more suitable framework for conceptual description. Another interesting and related issue is the effect of such frameworks on interpretation of evaluation data from prototyping activities which might be conducted as part of a human-centered design process. A further question to be addressed is the role of such frameworks in the context of broader methodologies for analysis and design.

\section{Discussion and Conclusions}

Analysis of our case study has revealed that the existing design frameworks probe different aspects of the work performed and suggest different forms of technology intervention. A question which arises is whether development teams should seek to adopt a single framework for a particular project, or whether it would be better to consider multiple points of view, starting from the same ethnographic data. Another question is whether there a case for "unifying" frameworks which bring together multiple design concepts, or is it sufficient to have a checklist of things to consider in design, which is derived from a variety of frameworks.

In conclusion, the decision of which conceptual framework to adopt should not be made lightly; ideally the development team should have a palette of concepts, from which they can choose according to the context. Designers should be wary of influence of the analysis framework on the design space. Furthermore, the relationship of the design framework with further stages of design process remains to be investigated.

\section{Acknowledgements}

J. McKnight is supported by an IRCSET/Intel Enterprise Partnership award.

\section{References}

1. Ash, J.S., Berg, M., Coiera, E.: Some Unintended Consequences of Information Technology in Health Care: The Nature of Patient Care Information System-related Errors. Journal of the American Medical Informatics Association 11(2), 104-112 (2004)

2. Bannon, L., Bødker, S.: Constructing Common Information Spaces. In: Proceedings of ECSCW 1997, pp. 81-96. Kluwer Academic Publishers, Norwell (1997)

3. Bardram, J.E., Bossen, C.: Mobility Work: The Spatial Dimension of Collaboration at a Hospital. Computer Supported Cooperative Work 14(2), 131-160 (2005)

4. Bardram, J.E., Bossen, C.: A Web of Coordinative Artifacts: Collaborative Work at a Hospital Ward. In: ACM Conference on Supporting Group Work. ACM Press, New York (2005)

5. Bossen, C.: The parameters of common information spaces: the heterogeneity of cooperative work at a hospital ward. In: Computer supported cooperative work. ACM Press, New York (2002) 
6. Christensen, H., Bardram, J.E.: Supporting Human Activities - Exploring ActivityCentered Computing. In: 4th International Conference on Ubiquitous Computing (2002)

7. Hartswood, M., Procter, R., Slack, R., Voß, A., Büscher, M., Rouncefield, M., Rouchy, P.: Co-realisation: towards a principled synthesis of ethnomethodology and participatory design. Scand. J. Inf. Syst. 14(2), 9-30 (2002)

8. Kane, B., Luz, S.: Multidisciplinary medical team meetings: An analysis of collaborative working with special attention to timing and teleconferencing. Computer Supported Cooperative Work 15(5), 501-535 (2006)

9. Nemeth, C., Cook, R.: Discovering and Supporting Temporal Cognition in Complex Environments. In: CogSci. 2004, Chicago, USA (2004)

10. Nemeth, C., Cook, R., et al.: The Messy Details: Insights from the Study of Technical Work in Healthcare. IEEE Trans. Syst. Man Cybern. 34(6), 689-692 (2004)

11. Reddy, M., Dourish, P., et al.: A Finger on the Pulse: Temporal Rhythms and Information Seeking in Medical Work. In: CSCW 2002, New Orleans, USA (2002)

12. Woods, D.D., Hollnagel, E.: Mapping Cognitive Demands in Complex Problem Solving Worlds. International Journal of Man Machine Studies 26, 257-275 (1987)

13. Reddy, M., Dourish, P., et al.: Coordinating Heterogeneous Work: information and Representation in Medical Care. In: European Conference on CSCW, Bonn, Germany (2001)

14. Nemeth, C., O'Connor, M., Klock, P., Cook, R.: Mapping Cognitive Work: The way out of IT System Failures. In: AMIA 2005 Annual Symposium, Washington, DC (2005) 